\title{
Housing Supply Elasticity and Rent Extraction by State and Local Governments Rebecca Diamond Online Appendix
}

\section{A Government Taxation under Income and Property Taxes}

In all the cases below I do not model the public sector labor market. This allows the analysis to focus on the role of the tax instrument in rent extraction.

\section{A Income Tax}

\section{A.1 Government}

The local government of city $j$ charges an income tax $\tau_{j}$ to workers who choose to reside within the city. The local government also produces government services, which cost $s_{j}$ for each worker in the city. $N_{j}$ measure the population of city $j$. The local rent seeking government maximizes:

$$
\max _{\tau_{j}, s_{j}} \tau_{j} w_{j} N_{j}-s_{j} N_{j}
$$

\section{A.2 Workers}

All workers are homogeneous. Workers living in city $j$ inelastically supply one unit of labor, and earn wage $w_{j}$. Each worker must rent a house to live in the city at rental rate $r_{j}$ and pay the local income tax $\tau_{j}$. Workers value the local amenities as measure by $A_{j}$. The desirability of government services $s_{j}$ is represented by $g\left(s_{j}\right)$. Thus, workers' utility from living in city $j$ is:

$$
U_{j}=w_{j}\left(1-\tau_{j}\right)-r_{j}+A_{j}+g\left(s_{j}\right)
$$

Workers maximize their utility by living in the city which they find the most desirable.

\section{A.3 Firms}

All firms are homogenous and produce a tradeable output $Y$. Cities exogenously differ in their productivity as measured by $\theta_{j}$. Local government services impact firms productivity, 
as measured by $b\left(s_{j}\right)$. The production function is:

$$
Y_{j}=\theta_{j} N_{j}+b\left(s_{j}\right) N_{j}
$$

I assume a completely elastic labor demand curve to focus on the role of housing supply elasticity in setting tax rates.

The labor market is perfectly competitive, so wages equal the marginal product of labor:

$$
w_{j}=\theta_{j}+b\left(s_{j}\right)
$$

\section{A.4 Housing}

The housing market is identical to the setting described in the main text in Section D. The housing supply curve is:

$$
\begin{aligned}
r_{j} & =a_{j}+\gamma_{j} \log \left(N_{j}\right) \\
\gamma_{j} & =\gamma x_{j}^{\text {house }}
\end{aligned}
$$

where $x_{j}^{\text {house }}$ is a vector of city characteristics which impact the elasticity of housing supply.

\section{A.5 Equilibrium in Labor and Housing}

Since all workers are identical, all cities with positive population must offer equal utility to workers. In equilibrium, all workers must be indifferent between all cities. Thus:

$$
U_{j}=w_{j}\left(1-\tau_{j}\right)-r_{j}+A_{j}+g\left(s_{j}\right)=\bar{U}
$$

Plugging in labor demand and housing supply gives:

$$
\left(\theta_{j}+b\left(s_{j}\right)\right)\left(1-\tau_{j}\right)-a_{j}-\gamma_{j} \log N_{j}+A_{j}+g\left(s_{j}\right)=\bar{U}
$$

Equation (11) determines the equilibrium distribution of workers across cities.

\section{A.6 Government Tax Competition}

The government maximizes:

$$
\max _{s_{j}, \tau_{j}} \tau_{j} w_{j} N_{j}-s_{j} N_{j}
$$


The first order conditions are:

$$
\begin{aligned}
0 & =w_{j} \tau_{j} \frac{\partial N_{j}}{\partial s_{j}}+\tau_{j} N_{j} \frac{\partial w_{j}}{\partial N_{j}} \frac{\partial N_{j}}{\partial s_{j}}-N_{j}-s_{j} \frac{\partial N_{j}}{\partial s_{j}} \\
0 & =\tau_{j}\left(\frac{\partial w_{j}}{\partial N_{j}} \frac{\partial N_{j}}{\partial \tau_{j}} N_{j}+w_{j} \frac{\partial N_{j}}{\partial \tau_{j}}\right)+w_{j} N_{j}-s_{j} \frac{\partial N_{j}}{\partial \tau_{j}} .
\end{aligned}
$$

Differentiating equation (11) to solve for $\frac{\partial N_{j}}{\partial s_{j}}$ and $\frac{\partial N_{j}}{\partial \tau_{j}}$ gives:

$$
\begin{aligned}
\frac{\partial N_{j}}{\partial s_{j}} & =N_{j} \frac{\left(1-\tau_{j}\right) b^{\prime}\left(s_{j}\right)+g^{\prime}\left(s_{j}\right)}{\gamma_{j}}>0 \\
\frac{\partial N_{j}}{\partial \tau_{j}} & =-N_{j} \frac{\left(\theta_{j}+b\left(s_{j}\right)\right)}{\gamma_{j}}<0 .
\end{aligned}
$$

Population increases with government services and decreases in taxes. Plugging these into (12) and combining the first order conditions shows that government services are provided such that the marginal benefit $\left(\left(1-\tau_{j}^{*}\right) b^{\prime}\left(s_{j}\right)+g^{\prime}\left(s_{j}\right)\right)$ equals marginal cost $(1)$ :

$$
\left(1-\tau_{j}^{*}\right) b^{\prime}\left(s_{j}^{*}\right)+g^{\prime}\left(s_{j}^{*}\right)=1
$$

This is the socially optimal level of government service, given the tax rate.

The equilibrium tax revenue per capita is:

$$
w_{j} \tau_{j}^{*}=\gamma_{j}+s_{j}^{*}
$$

To analyze the effect of housing supply elasticity on governments' ability to extract rent from taxes, I differentiate the tax markup with respect to the slope of the inverse housing supply curve, $\gamma_{j}$.

$$
\frac{\partial}{\partial \gamma_{j}}\left(w_{j} \tau_{j}^{*}-s_{j}^{*}\right)=1>0
$$

The government can extract more rent through higher taxes in a city with a less elastic housing supply with a income tax instrument. 


\section{B Property Tax}

\section{B.1 Government}

The local government of city $j$ charges a property tax $\tau_{j}$ to workers who choose to reside within the city. The local rent seeking government maximizes:

$$
\max _{\tau_{j}, s_{j}} \tau_{j} r_{j} N_{j}-s_{j} N_{j}
$$

\section{B.2 Workers}

Workers' utility from living in city $j$ facing a property tax $\tau_{j}$ is:

$$
U_{j}=w_{j}-r_{j}\left(1+\tau_{j}\right)+A_{j}+g\left(s_{j}\right)
$$

\section{B.3 Firms}

The production function is:

$$
Y_{j}=\theta_{j} N_{j}+b\left(s_{j}\right) N_{j}
$$

I assume a completely elastic labor demand curve to focus on the role of housing supply elasticity in setting tax rates.

The labor market is perfectly competitive, so wages equal the marginal product of labor:

$$
w_{j}=\theta_{j}+b\left(s_{j}\right)
$$

\section{B.4 Housing}

The housing market is identical to the setting described in the main text in Section D. The housing supply curve is:

$$
\begin{aligned}
r_{j} & =a_{j}+\gamma_{j} \log \left(N_{j}\right) \\
\gamma_{j} & =\gamma x_{j}^{\text {house }}
\end{aligned}
$$

where $x_{j}^{\text {house }}$ is a vector of city characteristics which impact the elasticity of housing supply. 


\section{B.5 Equilibrium in Labor and Housing}

Since all workers are identical, all cities with positive population must offer equal utility to workers. In equilibrium, all workers must be indifferent between all cities. Thus:

$$
U_{j}=w_{j}-r_{j}\left(1+\tau_{j}\right)+A_{j}+g\left(s_{j}\right)=\bar{U} .
$$

Plugging in labor demand and housing supply gives:

$$
\left(\theta_{j}+b\left(s_{j}\right)\right)-\left(a_{j}+\gamma_{j} \log N_{j}\right)\left(1+\tau_{j}\right)+A_{j}+g\left(s_{j}\right)=\bar{U}
$$

Equation (16a) determines the equilibrium distribution of workers across cities.

\section{B.6 Government Tax Competition}

The government maximizes:

$$
\max _{s_{j}, \tau_{j}} \tau_{j} r_{j} N_{j}-s_{j} N_{j}
$$

The first order conditions are:

$$
\begin{aligned}
0 & =r_{j} \tau_{j} \frac{\partial N_{j}}{\partial s_{j}}+\tau_{j} N_{j} \frac{\partial r_{j}}{\partial N_{j}} \frac{\partial N_{j}}{\partial s_{j}}-N_{j}-s_{j} \frac{\partial N_{j}}{\partial s_{j}} \\
0 & =\tau_{j}\left(\frac{\partial r_{j}}{\partial N_{j}} \frac{\partial N_{j}}{\partial \tau_{j}} N_{j}+r_{j} \frac{\partial N_{j}}{\partial \tau_{j}}\right)+r_{j} N_{j}-s_{j} \frac{\partial N_{j}}{\partial \tau_{j}} .
\end{aligned}
$$

Differentiating equation $(16 a)$ to solve for $\frac{\partial N_{j}}{\partial s_{j}}$ and $\frac{\partial N_{j}}{\partial \tau_{j}}$ gives:

$$
\begin{aligned}
\frac{\partial N_{j}}{\partial s_{j}} & =N_{j} \frac{b^{\prime}\left(s_{j}\right)+g^{\prime}\left(s_{j}\right)}{\gamma_{j}\left(1+\tau_{j}\right)}>0 \\
\frac{\partial N_{j}}{\partial \tau_{j}} & =-N_{j} \frac{r_{j}}{\gamma_{j}\left(1+\tau_{j}\right)}<0 .
\end{aligned}
$$

Combining the first order conditions shows that government services are provided such that the marginal benefit $\left(b^{\prime}\left(s_{j}\right)+g^{\prime}\left(s_{j}\right)\right)$ equals marginal cost $(1)$, which is the same finding for an income tax and head tax:

$$
b^{\prime}\left(s_{j}^{*}\right)+g^{\prime}\left(s_{j}^{*}\right)=1 .
$$

Plugging (19) into (18) and rearranging shows the equilibrium tax revenue per capita is:

$$
r_{j} \tau_{j}^{*}=\gamma_{j}+s_{j}^{*}
$$


Differentiating the tax markup with respect to the slope of the inverse housing supply curve, $\gamma_{j}$.

$$
\frac{\partial}{\partial \gamma_{j}}\left(w_{j} \tau_{j}^{*}-s_{j}^{*}\right)=1>0 .
$$

The government can extract more rent through higher taxes in a city with a less elastic housing supply using a property tax instrument. In the case of a property tax, as opposed to a head tax, there are four mechanisms through which a tax rate change impacts government revenue. To break these down, I rewrite the tax rate first order condition:

$$
0=\underbrace{\tau_{j} r_{j} \frac{\partial N_{j}}{\partial \tau_{j}}}_{\begin{array}{c}
\text { Decline in revenue due } \\
\text { to population decrease }
\end{array}}+\underbrace{\tau_{j} \frac{\partial r_{j}}{\partial N_{j}} \frac{\partial N_{j}}{\partial \tau_{j}} N_{j}}_{\begin{array}{c}
\text { Decline in revenue } \\
\text { due to rent decrease }
\end{array}}+\underbrace{r_{\text {from }}^{N_{j}}}_{\begin{array}{c}
\text { Additional tax revenue } \\
\text { from each resident }
\end{array}}-\underbrace{s_{j}^{\frac{\partial N_{j}}{\partial \tau_{j}}}}_{\begin{array}{c}
\text { Government services } \\
\text { cost savings }
\end{array}}
$$

First, the amount of out-migration driven by a tax hike is influenced by the local housing supply elasticity. This is the first term of equation (22). Second, the out-migration lowers rents and directly impacts tax revenues since the tax revenue is a percentage of housing rents. This is the second term of equation (22). However, the housing supply elasticity will not impact the size of the rental rate decrease in response to a tax hike. To see this, recall the equilibrium condition, equation (16a). For workers to derive utility $\bar{U}$ from this local area, the utility impact of a tax increase must be perfectly offset by a rent decrease. ${ }^{27}$ Thus, the equilibrium rental rate response to a given tax increase does not depend on the local housing supply elasticity. Indeed, the housing supply elasticity determines the migration response required to change housing rents in order to offset the utility impact of the tax increase. Thus, a more inelastic housing supply decreases the elasticity of government revenue with respect to the tax rate, giving the government more market power when using a property tax instrument.

The third and forth terms of equation (22) show a tax increase raises government revenues from each household and lowers the cost of government services due to out-migration. These channels also appear in the case of a head tax instrument.

\footnotetext{
${ }^{27}$ Since I have assumed a perfectly elastic labor demand curve, the rental rate response to a tax increase would be the same in any city. However, if labor demand was not perfectly elastic, then the rental rate response to a tax increase could differ with housing supply elasticity, since housing supply elasticity would influence the relative incidence of the tax rate on wages versus rents.
} 


\section{B Public Sector Compensation \& Employment by Type of Worker}

Appendix Table A.1 reports summary statistics on government payrolls, employment, FTEs, and wages spent on air transport, corrections, elementary \& secondary education, higher education, financial administration, fire protection, judicial \& legal, other government administration, health \& hospitals, housing \& community development, libraries, natural resources, parks \& recreation, police protection, public welfare, sanitation, water transport, utilities, and other spending not otherwise classified. Note that many counties do not have expenditures in every spending category. Thus, to retain the zeros in the data, regressions run on these data will be estimated in levels, not logs so that 0 spending and employment levels can be included in the regressions. All dollar amounts are deflated by the CPI-U and reported in constant 2000 dollars. $^{28}$

Table A.2 reports positive point estimates indicating less land availability raises government payrolls per county resident in 16 of the 19 categories of government spending when collective bargaining is prohibited. Eight of these estimates are statistically significant. In states outlawing bargaining, a one standard deviation increase in land unavailability increases monthly government payrolls per county resident by $\$ 14.66$ on corrections. Relative to counties' average monthly spending on corrections payroll per county resident of $\$ 132$, this represents an $11 \%$ increase. Similarly, financial administration payrolls increase by $8.6 \%$ (\$15.20, relative to a mean spending of $\$ 177)$, other government admin payrolls increase by $5.9 \%$ ( $\$ 12.50$, relative to a mean of $\$ 211$ ), housing and community development increase by $10.7 \%$, libraries increase by $21 \%$, parks and recreation increase by $11.2 \%$, sanitation increases by $5 \%$, and water transport increases by $159 \%$. Increased spending on parks \& recreation and water transport can likely be attributed directly to the topography in these areas, such as presence of bodies of water and other land features which would be likely be used as park areas. It is hard to offer a unifying reason of why the additional specific categories show statistically significant responses to land unavailability. Overall, many types of government spending appear to increase in land unavailable areas that have no collective bargaining provisions.

Government payrolls are significantly larger across many government categories in inelastic areas which allow public sector collective bargaining. Table A.2 shows 16 of the 19 categories have positive point estimates of the effect of land unavailability, with 11 of them being statistically significant. Land unavailability raises government payrolls broadly

\footnotetext{
${ }^{28}$ Regression analysis on elemetary \& secondary education, fire protection, and police protection use the public sector collective bargaining law data explicitly for teachers, fire fighters, and police, respectively.
} 
across many types of government spending all states, but significantly more when collective bargaining is legal.

Table A.3 shows whether these increased government payrolls led to wage increases for government employees. There appears to be no wage effects in states which prohibit collective bargaining. The point estimates for the wage effects across government categories are $50 \%$ positive, $50 \%$ negative, with only 2 estimates being significant at the $10 \%$ level, which is expected due to running 19 regressions.

In the states which allow collective bargaining, $100 \%$ of the point estimates show a positive wage response to land unavailability, with 17 of the 19 being statistically significant. Collective bargaining seems to channel these extra payroll dollars into higher government wages across essentially all types of government spending.

Table A.4 shows the effects of land unavailability on government FTEs per county resident. In states which prohibit collective bargaining, the categories which showed a significant positive government payroll response to land unavailability also show a statistically significant FTE per county resident response as well. These additional dollars all appear to go to a larger government workforce when collective bargaining is prohibited.

Within states which allow collective bargaining, government FTEs appear to statistically significantly rise in air transport, higher education, and parks \& recreation. FTEs fall in elementary \& secondary education, and libraries. The other point estimates are a mix of both positive and negative effects. There does not appear to be a clear increase in FTEs in collective bargaining states. Table A.5 looks at government employment counts per county resident and shows very similar results as those for the government FTEs. 
Table A.1: Summary Statistics for County Area Government Employment and Payroll by Type of Worker

Monthly Gov Worker Payroll Gov Worker Employment per Gov Worker FTE per 100

\begin{tabular}{|c|c|c|c|c|c|c|c|c|c|c|c|c|}
\hline & \multicolumn{3}{|c|}{$\begin{array}{l}\text { Monthly Gov Worker Payroll } \\
\text { per } 100 \text { Residents }\end{array}$} & \multicolumn{3}{|c|}{$\begin{array}{l}\text { Gov Worker Employment per } \\
100 \text { Residents }\end{array}$} & \multicolumn{3}{|c|}{$\begin{array}{c}\text { Gov Worker FTE per } 100 \\
\text { Residents }\end{array}$} & \multicolumn{3}{|c|}{ Ln Average Gov Worker Wage } \\
\hline & Obs & Mean & Std. Dev. & Obs & Mean & Std. Dev. & Obs & Mean & Std. Dev. & Obs & Mean & Std. Dev. \\
\hline County Area Overall & 6069 & 9275.80 & 3142.16 & 6069 & 4.02 & 1.06 & 6069 & 3.41 & 0.90 & 6069 & 8.89 & 0.18 \\
\hline Air Transport & 6069 & 19.67 & 89.11 & 6069 & 0.01 & 0.02 & 6069 & 0.01 & 0.02 & 2528 & 7.88 & 0.30 \\
\hline Correction & 6069 & 132.07 & 141.04 & 6069 & 0.05 & 0.05 & 6069 & 0.05 & 0.05 & 5250 & 7.77 & 0.29 \\
\hline \multicolumn{13}{|l|}{ Elementary \& } \\
\hline Secondary Education & 6069 & 5518.47 & 1825.90 & 6069 & 2.32 & 0.69 & 6069 & 2.01 & 0.60 & 6045 & 7.91 & 0.19 \\
\hline Higher Education & 6069 & 209.43 & 411.07 & 6069 & 0.11 & 0.22 & 6069 & 0.06 & 0.13 & 1779 & 8.10 & 0.20 \\
\hline Finacial Admin & 6069 & 177.40 & 92.89 & 6069 & 0.08 & 0.04 & 6069 & 0.07 & 0.03 & 6058 & 7.81 & 0.24 \\
\hline Fire Protection & 6069 & 267.40 & 244.02 & 6069 & 0.11 & 0.08 & 6069 & 0.08 & 0.06 & 5535 & 7.99 & 0.31 \\
\hline Judicial \& Legal & 6069 & 126.34 & 140.21 & 6069 & 0.05 & 0.05 & 6069 & 0.05 & 0.05 & 4368 & 7.88 & 0.31 \\
\hline Other Gov Admin & 6069 & 211.04 & 125.20 & 6069 & 0.13 & 0.09 & 6069 & 0.08 & 0.04 & 6055 & 7.86 & 0.26 \\
\hline Health \& Hospitals & 6069 & 567.17 & 936.87 & 6069 & 0.26 & 0.40 & 6069 & 0.23 & 0.36 & 5530 & 7.79 & 0.26 \\
\hline \multicolumn{13}{|l|}{ Housing \& Community } \\
\hline Development & 6069 & 63.46 & 84.36 & 6069 & 0.03 & 0.03 & 6069 & 0.02 & 0.03 & 4709 & 7.83 & 0.26 \\
\hline Libraries & 6069 & 67.68 & 92.42 & 6069 & 0.05 & 0.07 & 6069 & 0.03 & 0.06 & 5004 & 7.62 & 0.27 \\
\hline Natural Resources & 6069 & 30.20 & 96.01 & 6069 & 0.01 & 0.04 & 6069 & 0.01 & 0.03 & 4680 & 7.67 & 0.36 \\
\hline Parks \& Recreation & 6069 & 117.17 & 119.73 & 6069 & 0.08 & 0.09 & 6069 & 0.05 & 0.05 & 5564 & 7.67 & 0.25 \\
\hline Police Protection & 6069 & 595.46 & 349.78 & 6069 & 0.22 & 0.09 & 6069 & 0.20 & 0.08 & 6058 & 7.94 & 0.26 \\
\hline Public Welfare & 6069 & 180.42 & 245.63 & 6069 & 0.08 & 0.12 & 6069 & 0.08 & 0.11 & 4811 & 7.68 & 0.29 \\
\hline Sanitation & 6069 & 181.96 & 135.87 & 6069 & 0.08 & 0.05 & 6069 & 0.07 & 0.05 & 5922 & 7.79 & 0.26 \\
\hline Water Transport & 6069 & 5.73 & 33.79 & 6069 & 0.00 & 0.01 & 6069 & 0.00 & 0.01 & 681 & 7.99 & 0.36 \\
\hline Other NEC & 6069 & 252.84 & 261.69 & 6069 & 0.14 & 0.16 & 6069 & 0.10 & 0.10 & 6034 & 7.75 & 0.26 \\
\hline Utilities & 6069 & 278.58 & 445.27 & 6069 & 0.10 & 0.12 & 6069 & 0.09 & 0.11 & 5790 & 7.88 & 0.25 \\
\hline
\end{tabular}

Notes: Data from Census of Governments cover 1972-2007 reported in five year intervals in years ending in 2 and 7. All dollar amounts are deflated by CPI-U to constant 2000 dollars. 
Table A.2: County Area Government Payroll per 100 Residents by Category vs. Housing Supply Elasticity Interactions

\begin{tabular}{|c|c|c|c|c|c|c|c|c|c|c|}
\hline & [1] & {$[2]$} & [3] & [4] & [5] & {$[6]$} & [7] & [8] & [9] & [10] \\
\hline & $\begin{array}{c}\text { Monthly Gov } \\
\text { Pay/100 } \\
\text { Residents }\end{array}$ & Air Transport & Correction & $\begin{array}{c}\text { Elementary } \\
\text { \& Secondary } \\
\text { Education }\end{array}$ & $\begin{array}{c}\text { Higher } \\
\text { Education }\end{array}$ & $\begin{array}{l}\text { Finacial } \\
\text { Admin }\end{array}$ & $\begin{array}{c}\text { Fire } \\
\text { Protection }\end{array}$ & $\begin{array}{c}\text { Judicial \& } \\
\text { Legal }\end{array}$ & $\begin{array}{c}\text { Other Gov } \\
\text { Admin }\end{array}$ & $\begin{array}{l}\text { Health \& } \\
\text { Hospitals }\end{array}$ \\
\hline \multicolumn{11}{|l|}{ Land } \\
\hline Unavailability & $\begin{array}{c}127.1 \\
{[165.6]}\end{array}$ & $\begin{array}{l}-1.969 \\
{[2.183]}\end{array}$ & $\begin{array}{l}14.66^{*} \\
{[7.920]}\end{array}$ & $\begin{array}{c}113.3 \\
{[123.1]}\end{array}$ & $\begin{array}{l}-15.49 \\
{[17.02]}\end{array}$ & $\begin{array}{c}15.20^{* *} \\
{[6.849]}\end{array}$ & $\begin{array}{c}12.27 \\
{[20.66]}\end{array}$ & $\begin{array}{c}5.248 \\
{[8.432]}\end{array}$ & $\begin{array}{l}12.50^{*} \\
{[7.050]}\end{array}$ & $\begin{array}{l}-57.27 \\
{[50.98]}\end{array}$ \\
\hline \multicolumn{11}{|l|}{ Collective } \\
\hline Bargainings & $\begin{array}{c}1279.1^{* * *} \\
{[260.0]}\end{array}$ & $\begin{array}{c}0.659 \\
{[4.356]}\end{array}$ & $\begin{array}{c}36.14 * * * \\
{[9.952]}\end{array}$ & $\begin{array}{c}612.0 * * * \\
{[136.9]}\end{array}$ & $\begin{array}{c}157.9 * * * \\
{[28.41]}\end{array}$ & $\begin{array}{l}14.05^{*} \\
{[8.455]}\end{array}$ & $\begin{array}{l}33.07^{*} \\
{[19.47]}\end{array}$ & $\begin{array}{c}49.12 * * * \\
{[9.216]}\end{array}$ & $\begin{array}{c}61.51 * * * \\
{[10.37]}\end{array}$ & $\begin{array}{l}-64.28 \\
{[68.30]}\end{array}$ \\
\hline \multicolumn{11}{|c|}{ Bargain*Unavai } \\
\hline lability & $\begin{array}{l}470.0^{*} \\
{[254.6]}\end{array}$ & $\begin{array}{c}10.05^{* *} \\
{[4.080]}\end{array}$ & $\begin{array}{l}21.78^{*} \\
{[12.22]}\end{array}$ & $\begin{array}{l}-111.7 \\
{[141.8]}\end{array}$ & $\begin{array}{c}68.93^{* *} \\
{[27.67]}\end{array}$ & $\begin{array}{l}14.28^{*} \\
{[7.817]}\end{array}$ & $\begin{array}{l}\text { 41.59* } \\
{[23.44]}\end{array}$ & $\begin{array}{c}16.1 \\
{[11.72]}\end{array}$ & $\begin{array}{l}20.64^{*} \\
{[11.95]}\end{array}$ & $\begin{array}{c}82.16 \\
{[69.57]}\end{array}$ \\
\hline Constant & $\begin{array}{c}7629.4^{* * *} \\
{[161.38]}\end{array}$ & $\begin{array}{c}9.870 * * * \\
{[2.119]}\end{array}$ & $\begin{array}{c}29.24 * * * \\
{[4.769]}\end{array}$ & $\begin{array}{c}4,838 * * * \\
{[114.5]}\end{array}$ & $\begin{array}{c}83.21 * * * \\
{[19.34]}\end{array}$ & $\begin{array}{c}142.1^{* * *} \\
{[6.198]}\end{array}$ & $\begin{array}{c}208.1^{* * *} \\
{[12.19]}\end{array}$ & $\begin{array}{c}-24.61 * * * \\
{[5.940]}\end{array}$ & $\begin{array}{c}226.5^{* * *} \\
{[7.924]}\end{array}$ & $\begin{array}{c}484.5^{* * *} \\
{[49.43]}\end{array}$ \\
\hline Observations & 6,069 & 6,069 & 6,069 & 6,069 & 6,069 & 6,069 & 6,069 & 6,069 & 6,069 & 6,069 \\
\hline \multirow[t]{3}{*}{ R-squared } & 0.16 & 0.01 & 0.254 & 0.107 & 0.048 & 0.153 & 0.065 & 0.374 & 0.21 & 0.007 \\
\hline & [11] & [12] & {$[13]$} & {$[14]$} & {$[15]$} & {$[16]$} & {$[17]$} & {$[18$} & [19] & {$[20]$} \\
\hline & $\begin{array}{l}\text { Housing \& } \\
\text { Community } \\
\text { Develop } \\
\end{array}$ & Libraries & $\begin{array}{c}\text { Natural } \\
\text { Resources } \\
\end{array}$ & $\begin{array}{c}\text { Parks \& } \\
\text { Recreation }\end{array}$ & $\begin{array}{c}\text { Police } \\
\text { Protection } \\
\end{array}$ & $\begin{array}{c}\text { Public } \\
\text { Welfare } \\
\end{array}$ & Sanitation & $\begin{array}{c}\text { Water } \\
\text { Transport }\end{array}$ & Other NEC & Utilities \\
\hline Land & & & & & & & & & & \\
\hline Unavailability & $\begin{array}{c}6.791^{* *} \\
{[2.740]}\end{array}$ & $\begin{array}{c}14.08^{* *} \\
{[7.037]}\end{array}$ & $\begin{array}{c}7.922 \\
{[5.730]}\end{array}$ & $\begin{array}{l}13.08^{*} \\
{[6.851]}\end{array}$ & $\begin{array}{c}12.89 \\
{[16.08]}\end{array}$ & $\begin{array}{c}15.37 \\
{[16.80]}\end{array}$ & $\begin{array}{l}9.014^{*} \\
{[4.882]}\end{array}$ & $\begin{array}{c}9.153^{* *} \\
{[4.554]}\end{array}$ & $\begin{array}{c}12.06 \\
{[11.63]}\end{array}$ & $\begin{array}{c}8.319 \\
{[16.02]}\end{array}$ \\
\hline Collective & & & & & & & & & & \\
\hline Bargainings & $\begin{array}{l}8.216^{*} \\
{[4.338]}\end{array}$ & $\begin{array}{l}14.66^{*} \\
{[7.838]}\end{array}$ & $\begin{array}{c}11.19 * * \\
{[5.549]}\end{array}$ & $\begin{array}{c}14.85 \\
{[10.41]}\end{array}$ & $\begin{array}{c}93.63 * * * \\
{[29.26]}\end{array}$ & $\begin{array}{c}124.1 * * * \\
{[25.00]}\end{array}$ & $\begin{array}{c}-1.444 \\
{[8.403]}\end{array}$ & $\begin{array}{l}-1.306 \\
{[3.211]}\end{array}$ & $\begin{array}{c}20.56 \\
{[15.29]}\end{array}$ & $\begin{array}{c}40.16 \\
{[28.46]}\end{array}$ \\
\hline Bargain*Unava & & & & & & & & & & \\
\hline lability & $\begin{array}{l}\text { 6.999* } \\
{[4.029]}\end{array}$ & $\begin{array}{l}-9.442 \\
{[7.862]}\end{array}$ & $\begin{array}{c}2.263 \\
{[5.467]}\end{array}$ & $\begin{array}{c}23.89 * * * \\
{[8.965]}\end{array}$ & $\begin{array}{c}94.63 * * * \\
{[28.93]}\end{array}$ & $\begin{array}{c}18.85 \\
{[22.30]}\end{array}$ & $\begin{array}{c}16.73 * * \\
{[7.786]}\end{array}$ & $\begin{array}{l}-2.404 \\
{[4.309]}\end{array}$ & $\begin{array}{c}38.59 * * \\
{[15.15]}\end{array}$ & $\begin{array}{c}35.63 \\
{[30.93]}\end{array}$ \\
\hline Constant & $\begin{array}{c}41.95^{* * *} \\
{[2.801]}\end{array}$ & $\begin{array}{c}41.52 * * * \\
{[5.014]}\end{array}$ & $\begin{array}{c}25.92 * * * \\
{[4.378]}\end{array}$ & $\begin{array}{c}83.97 * * * \\
{[6.966]}\end{array}$ & $\begin{array}{c}393.1 * * * \\
{[16.03]}\end{array}$ & $\begin{array}{c}75.65^{* * *} \\
{[11.81]}\end{array}$ & $\begin{array}{c}162.7^{* * *} \\
{[5.969]}\end{array}$ & $\begin{array}{c}8.328 * * * \\
{[2.712]}\end{array}$ & $\begin{array}{c}212.5^{* * *} \\
{[10.83]}\end{array}$ & $\begin{array}{c}197.5^{* * *} \\
{[17.73]}\end{array}$ \\
\hline Observations & 6,069 & 6,069 & 6,069 & 6,069 & 6,069 & 6,069 & 6,069 & 6,069 & 6,069 & 6,069 \\
\hline R-squared & 0.035 & 0.06 & 0.013 & 0.082 & 0.178 & 0.091 & 0.026 & 0.045 & 0.056 & 0.012 \\
\hline
\end{tabular}

Notes: Standard errors clustered by MSA. Data on monthly government payroll per capita is measured in constast 2000 dollars. Counties with zero spending in a given

category are included in regressions. Controls include year dummies. ${ }^{* * *} p<0.01,{ }^{* *} p<0.05,{ }^{*} p<0.1$ 
Table A.3: County Area Average Gov Wage by Category vs. Housing Supply Elasticity Interactions

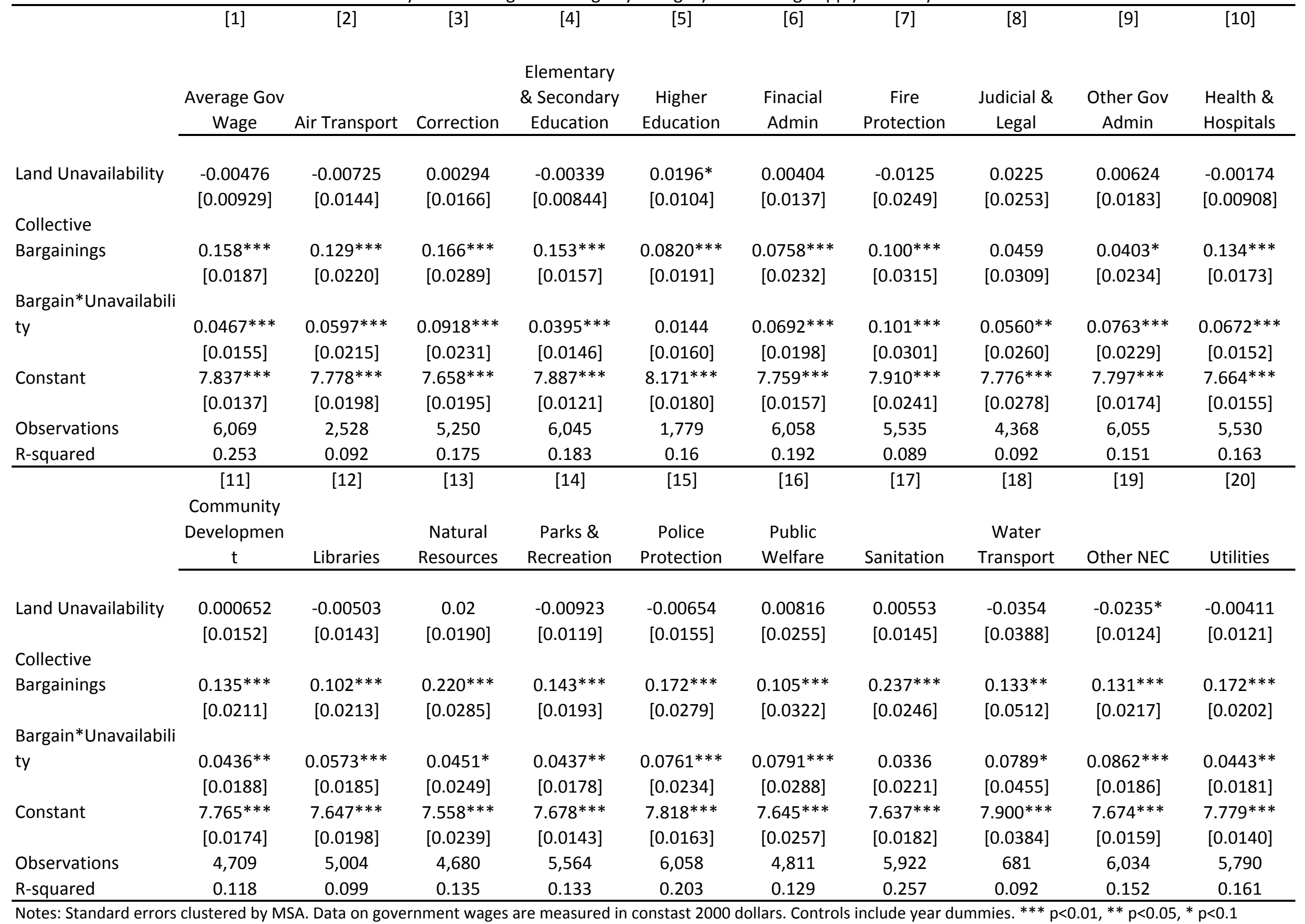


Table A.4: County Area FTE Gov Workers per 100 Residents by Category vs. Housing Supply Elasticity Interactions

\begin{tabular}{|c|c|c|c|c|c|c|c|c|c|c|}
\hline & {$[1]$} & {$[2]$} & [3] & {$[4]$} & [5] & [6] & [7] & [8] & [9] & [10] \\
\hline & $\begin{array}{l}\text { FTE Gov/100 } \\
\text { Residents }\end{array}$ & Air Transport & Correction & $\begin{array}{l}\text { Elementary } \\
\text { \& Secondary } \\
\text { Education }\end{array}$ & $\begin{array}{c}\text { Higher } \\
\text { Education }\end{array}$ & $\begin{array}{l}\text { Finacial } \\
\text { Admin }\end{array}$ & $\begin{array}{c}\text { Fire } \\
\text { Protection }\end{array}$ & $\begin{array}{c}\text { Judicial \& } \\
\text { Legal }\end{array}$ & $\begin{array}{c}\text { Other Gov } \\
\text { Admin }\end{array}$ & $\begin{array}{l}\text { Health \& } \\
\text { Hospitals }\end{array}$ \\
\hline \multicolumn{11}{|l|}{ Land } \\
\hline Unavailability & $\begin{array}{c}0.0696 \\
{[0.0530]}\end{array}$ & $\begin{array}{c}-0.000288 \\
{[0.000635]}\end{array}$ & $\begin{array}{l}0.00661 * \\
{[0.00364]}\end{array}$ & $\begin{array}{c}0.0575 \\
{[0.0445]}\end{array}$ & $\begin{array}{c}-0.00662 \\
{[0.00562]}\end{array}$ & $\begin{array}{c}0.00597 * * * \\
{[0.00200]}\end{array}$ & $\begin{array}{c}0.00614 \\
{[0.00580]}\end{array}$ & $\begin{array}{c}0.00173 \\
{[0.00388]}\end{array}$ & $\begin{array}{l}0.00378 * \\
{[0.00219]}\end{array}$ & $\begin{array}{c}-0.027 \\
{[0.0202]}\end{array}$ \\
\hline \multicolumn{11}{|l|}{ Collective } \\
\hline Bargainings & $\begin{array}{c}-0.0952 \\
{[0.0594]}\end{array}$ & $\begin{array}{c}-0.000265 \\
{[0.00104]}\end{array}$ & $\begin{array}{c}0.00395 \\
{[0.00332]}\end{array}$ & $\begin{array}{c}-0.0863 * * \\
{[0.0395]}\end{array}$ & $\begin{array}{c}0.0429 * * * \\
{[0.00875]}\end{array}$ & $\begin{array}{c}0.00061 \\
{[0.00232]}\end{array}$ & $\begin{array}{r}-0.000332 \\
{[0.00480]}\end{array}$ & $\begin{array}{c}0.0141 * * * \\
{[0.00339]}\end{array}$ & $\begin{array}{c}0.0187^{* * *} \\
{[0.00279]}\end{array}$ & $\begin{array}{c}-0.0535^{* *} \\
{[0.0271]}\end{array}$ \\
\hline \multicolumn{11}{|l|}{ Bargain* } \\
\hline Unavailability & $\begin{array}{c}-0.0279 \\
{[0.0600]}\end{array}$ & $\begin{array}{l}0.00222 * * \\
{[0.000979]}\end{array}$ & $\begin{array}{c}0.000153 \\
{[0.00427]}\end{array}$ & $\begin{array}{c}-0.131 * * * \\
{[0.0465]}\end{array}$ & $\begin{array}{c}0.0181 * * \\
{[0.00806]}\end{array}$ & $\begin{array}{c}-0.000822 \\
{[0.00219]}\end{array}$ & $\begin{array}{c}0.000661 \\
{[0.00591]}\end{array}$ & $\begin{array}{c}0.0000124 \\
{[0.00414]}\end{array}$ & $\begin{array}{r}-0.000823 \\
{[0.00309]}\end{array}$ & $\begin{array}{c}0.0125 \\
{[0.0256]}\end{array}$ \\
\hline Constant & $\begin{array}{c}2.986 * * * \\
{[0.0441]}\end{array}$ & $\begin{array}{c}0.00401 * * * \\
{[0.000556]}\end{array}$ & $\begin{array}{c}0.0160 * * * \\
{[0.00192]}\end{array}$ & $\begin{array}{c}1.801 * * * \\
{[0.0378]}\end{array}$ & $\begin{array}{c}0.0219 * * * \\
{[0.00606]}\end{array}$ & $\begin{array}{c}0.0595^{* * *} \\
{[0.00197]}\end{array}$ & $\begin{array}{c}0.0721 * * * \\
{[0.00374]}\end{array}$ & $\begin{array}{c}-0.00719 * * * \\
{[0.00220]}\end{array}$ & $\begin{array}{c}0.0900 * * * \\
{[0.00256]}\end{array}$ & $\begin{array}{c}0.240 * * * \\
{[0.0223]}\end{array}$ \\
\hline Observations & 6,069 & 6,069 & 6,069 & 6,069 & 6,069 & 6,069 & 6,069 & 6,069 & 6,069 & 6,069 \\
\hline \multirow[t]{3}{*}{ R-squared } & 0.12 & 0.01 & 0.226 & 0.125 & 0.037 & 0.057 & 0.027 & 0.388 & 0.199 & 0.011 \\
\hline & {$[11]$} & [12] & {$[13]$} & {$[14]$} & {$[15]$} & {$[16]$} & {$[17]$} & {$[18]$} & [19] & {$[20]$} \\
\hline & $\begin{array}{c}\text { Housing \& } \\
\text { Community } \\
\text { Development }\end{array}$ & Libraries & $\begin{array}{c}\text { Natural } \\
\text { Resources }\end{array}$ & $\begin{array}{c}\text { Parks \& } \\
\text { Recreation }\end{array}$ & $\begin{array}{c}\text { Police } \\
\text { Protection }\end{array}$ & $\begin{array}{c}\text { Public } \\
\text { Welfare }\end{array}$ & Sanitation & $\begin{array}{c}\text { Water } \\
\text { Transport }\end{array}$ & Other NEC & Utilities \\
\hline \multicolumn{11}{|l|}{ Land } \\
\hline Unavailability & $\begin{array}{c}0.00287 * * * \\
{[0.00103]}\end{array}$ & $\begin{array}{c}0.00713 * * \\
{[0.00291]}\end{array}$ & $\begin{array}{c}0.00327 \\
{[0.00256]}\end{array}$ & $\begin{array}{c}0.00702 * * \\
{[0.00288]}\end{array}$ & $\begin{array}{c}0.00828 \\
{[0.00558]}\end{array}$ & $\begin{array}{c}0.00722 \\
{[0.00660]}\end{array}$ & $\begin{array}{c}0.00386 * * \\
{[0.00176]}\end{array}$ & $\begin{array}{l}0.00349 * \\
{[0.00178]}\end{array}$ & $\begin{array}{c}0.00837^{*} \\
{[0.00476]}\end{array}$ & $\begin{array}{c}0.00416 \\
{[0.00550]}\end{array}$ \\
\hline \multicolumn{11}{|l|}{ Collective } \\
\hline Bargainings & $\begin{array}{r}-0.000587 \\
{[0.00154]}\end{array}$ & $\begin{array}{l}0.00580 * \\
{[0.00345]}\end{array}$ & $\begin{array}{c}0.00188 \\
{[0.00209]}\end{array}$ & $\begin{array}{c}-0.000793 \\
{[0.00401]}\end{array}$ & $\begin{array}{c}-0.00557 \\
{[0.00585]}\end{array}$ & $\begin{array}{c}0.0497 * * * \\
{[0.0101]}\end{array}$ & $\begin{array}{c}-0.0175 * * * \\
{[0.00298]}\end{array}$ & $\begin{array}{c}-0.00101 \\
{[0.00118]}\end{array}$ & $\begin{array}{l}-0.00516 \\
{[0.00524]}\end{array}$ & $\begin{array}{c}-0.00738 \\
{[0.00752]}\end{array}$ \\
\hline \multicolumn{11}{|l|}{ Bargain* } \\
\hline Unavailability & $\begin{array}{l}0.000751 \\
{[0.00132]}\end{array}$ & $\begin{array}{c}-0.00666 * * \\
{[0.00336]}\end{array}$ & $\begin{array}{c}-0.000597 \\
{[0.00205]}\end{array}$ & $\begin{array}{l}0.00656^{*} \\
{[0.00339]}\end{array}$ & $\begin{array}{c}0.00838 \\
{[0.00704]}\end{array}$ & $\begin{array}{c}-0.000636 \\
{[0.00854]}\end{array}$ & $\begin{array}{c}0.00326 \\
{[0.00284]}\end{array}$ & $\begin{array}{c}-0.00159 \\
{[0.00159]}\end{array}$ & $\begin{array}{c}0.00446 \\
{[0.00567]}\end{array}$ & $\begin{array}{c}0.00485 \\
{[0.00767]}\end{array}$ \\
\hline Constant & $\begin{array}{c}0.0180 * * * \\
{[0.00120]}\end{array}$ & $\begin{array}{c}0.0180 * * * \\
{[0.00211]}\end{array}$ & $\begin{array}{c}0.0123 * * * \\
{[0.00193]}\end{array}$ & $\begin{array}{c}0.0384 * * * \\
{[0.00280]}\end{array}$ & $\begin{array}{l}0.154 * * * \\
{[0.00384]}\end{array}$ & $\begin{array}{c}0.0337 * * * \\
{[0.00470]}\end{array}$ & $\begin{array}{c}0.0783 * * * \\
{[0.00241]}\end{array}$ & $\begin{array}{c}0.00290 * * * \\
{[0.000954]}\end{array}$ & $\begin{array}{c}0.0943 * * * \\
{[0.00439]}\end{array}$ & $\begin{array}{c}0.0805^{* * *} \\
{[0.00583]}\end{array}$ \\
\hline Observations & 6,069 & 6,069 & 6,069 & 6,069 & 6,069 & 6,069 & 6,069 & 6,069 & 6,069 & 6,069 \\
\hline R-squared & 0.023 & 0.034 & 0.008 & 0.068 & 0.134 & 0.069 & 0.04 & 0.042 & 0.051 & 0.01 \\
\hline
\end{tabular}


Table A.5: County Area Gov Worker Employment per 100 Residents by Category vs. Housing Supply Elasticity Interactions

$\left[\begin{array}{llllllll}1 & {[2]} & {[3]} & {[4]} & {[5]} & {[6]} & {[7]} & {[8]}\end{array}\right]$

[10]

\begin{tabular}{|c|c|c|c|c|c|c|c|c|c|c|}
\hline & $\begin{array}{c}\text { Gov Emp/100 } \\
\text { Residents }\end{array}$ & Air Transport & Correction & $\begin{array}{c}\text { Elementary } \\
\text { \& Secondary } \\
\text { Education }\end{array}$ & $\begin{array}{c}\text { Higher } \\
\text { Education }\end{array}$ & $\begin{array}{l}\text { Finacial } \\
\text { Admin }\end{array}$ & $\begin{array}{c}\text { Fire } \\
\text { Protection }\end{array}$ & $\begin{array}{c}\text { Judicial \& } \\
\text { Legal }\end{array}$ & $\begin{array}{c}\text { Other Gov } \\
\text { Admin }\end{array}$ & $\begin{array}{l}\text { Health \& } \\
\text { Hospitals }\end{array}$ \\
\hline \multicolumn{11}{|l|}{ Land } \\
\hline Unavailability & $\begin{array}{c}0.0809 \\
{[0.0549]}\end{array}$ & $\begin{array}{l}-0.000292 \\
{[0.000661]}\end{array}$ & $\begin{array}{l}0.00662^{*} \\
{[0.00368]}\end{array}$ & $\begin{array}{c}0.072 \\
{[0.0504]}\end{array}$ & $\begin{array}{c}-0.0106 \\
{[0.00994]}\end{array}$ & $\begin{array}{c}0.00516^{* *} \\
{[0.00214]}\end{array}$ & $\begin{array}{c}0.00271 \\
{[0.00624]}\end{array}$ & $\begin{array}{c}0.00127 \\
{[0.00422]}\end{array}$ & $\begin{array}{c}0.00102 \\
{[0.00276]}\end{array}$ & $\begin{array}{l}-0.0304 \\
{[0.0223]}\end{array}$ \\
\hline \multicolumn{11}{|l|}{ Collective } \\
\hline Bargainings & $\begin{array}{c}0.1419 * * \\
{[0.0697]}\end{array}$ & $\begin{array}{l}-0.000183 \\
{[0.00109]}\end{array}$ & $\begin{array}{c}0.00551 \\
{[0.00341]}\end{array}$ & $\begin{array}{l}-0.0574 \\
{[0.0458]}\end{array}$ & $\begin{array}{c}0.0798 * * * \\
{[0.0153]}\end{array}$ & $\begin{array}{c}0.0104^{* * *} \\
{[0.00274]}\end{array}$ & $\begin{array}{c}0.0167^{* * *} \\
{[0.00575]}\end{array}$ & $\begin{array}{c}0.0174 * * * \\
{[0.00371]}\end{array}$ & $\begin{array}{c}0.0541 * * * \\
{[0.00529]}\end{array}$ & $\begin{array}{l}-0.0509 * \\
{[0.0300]}\end{array}$ \\
\hline \multicolumn{11}{|l|}{ Bargain* } \\
\hline Unavailability & $\begin{array}{l}-0.0863 \\
{[0.0678]}\end{array}$ & $\begin{array}{c}0.00211^{* *} \\
{[0.00100]}\end{array}$ & $\begin{array}{l}-0.000227 \\
{[0.00436]}\end{array}$ & $\begin{array}{c}-0.141 * * * \\
{[0.0511]}\end{array}$ & $\begin{array}{c}0.0352 * * \\
{[0.0149]}\end{array}$ & $\begin{array}{l}-0.00302 \\
{[0.00252]}\end{array}$ & $\begin{array}{l}-0.00124 \\
{[0.00640]}\end{array}$ & $\begin{array}{l}-0.000495 \\
{[0.00452]}\end{array}$ & $\begin{array}{c}-0.0159 * * * \\
{[0.00450]}\end{array}$ & $\begin{array}{l}0.00942 \\
{[0.0285]}\end{array}$ \\
\hline Constant & $\begin{array}{c}3.4391 * * * \\
{[0.0486]}\end{array}$ & $\begin{array}{c}0.00433^{* * *} \\
{[0.000584]}\end{array}$ & $\begin{array}{c}0.0165 * * * \\
{[0.00197]}\end{array}$ & $\begin{array}{c}2.076 * * * \\
{[0.0425]}\end{array}$ & $\begin{array}{c}0.0347 * * * \\
{[0.0107]}\end{array}$ & $\begin{array}{c}0.0690 * * * \\
{[0.00204]}\end{array}$ & $\begin{array}{l}0.100 * * * \\
{[0.00559]}\end{array}$ & $\begin{array}{c}-0.00901 * * * \\
{[0.00243]}\end{array}$ & $\begin{array}{l}0.119 * * * \\
{[0.00429]}\end{array}$ & $\begin{array}{c}0.256 * * * \\
{[0.0242]}\end{array}$ \\
\hline Observations & 6,069 & 6,069 & 6,069 & 6,069 & 6,069 & 6,069 & 6,069 & 6,069 & 6,069 & 6,069 \\
\hline \multirow[t]{3}{*}{ R-squared } & 0.11 & 0.01 & 0.224 & 0.102 & 0.041 & 0.059 & 0.021 & 0.405 & 0.196 & 0.01 \\
\hline & [11] & {$[12]$} & {$[13]$} & {$[14]$} & {$[15]$} & {$[16]$} & {$[17]$} & {$[18]$} & {$[19]$} & {$[20]$} \\
\hline & $\begin{array}{c}\text { Housing \& } \\
\text { Community } \\
\text { Development }\end{array}$ & Libraries & $\begin{array}{c}\text { Natural } \\
\text { Resources }\end{array}$ & $\begin{array}{c}\text { Parks \& } \\
\text { Recreation }\end{array}$ & $\begin{array}{c}\text { Police } \\
\text { Protection } \\
\end{array}$ & $\begin{array}{c}\text { Public } \\
\text { Welfare }\end{array}$ & Sanitation & $\begin{array}{c}\text { Water } \\
\text { Transport }\end{array}$ & Other NEC & Utilities \\
\hline Land & & & & & & & & & & \\
\hline Unavailability & $\begin{array}{c}0.00262 * * \\
{[0.00109]}\end{array}$ & $\begin{array}{c}0.00854^{* *} \\
{[0.00413]}\end{array}$ & $\begin{array}{c}0.00328 \\
{[0.00255]}\end{array}$ & $\begin{array}{l}0.0120 * * \\
{[0.00526]}\end{array}$ & $\begin{array}{c}0.00793 \\
{[0.00595]}\end{array}$ & $\begin{array}{c}0.00805 \\
{[0.00707]}\end{array}$ & $\begin{array}{c}0.00392 * * \\
{[0.00181]}\end{array}$ & $\begin{array}{c}0.00363 * * \\
{[0.00183]}\end{array}$ & $\begin{array}{c}0.00906 \\
{[0.00589]}\end{array}$ & $\begin{array}{c}0.00445 \\
{[0.00586]}\end{array}$ \\
\hline Collective & & & & & & & & & & \\
\hline Bargainings & $\begin{array}{l}-0.000371 \\
{[0.00160]}\end{array}$ & $\begin{array}{l}0.0117^{* *} \\
{[0.00526]}\end{array}$ & $\begin{array}{c}0.00183 \\
{[0.00216]}\end{array}$ & $\begin{array}{c}0.00576 \\
{[0.00717]}\end{array}$ & $\begin{array}{c}0.0038 \\
{[0.00641]}\end{array}$ & $\begin{array}{c}0.0541 * * * \\
{[0.0109]}\end{array}$ & $\begin{array}{c}-0.0159 * * * \\
{[0.00306]}\end{array}$ & $\begin{array}{l}-0.000949 \\
{[0.00123]}\end{array}$ & $\begin{array}{c}0.0223^{* * *} \\
{[0.00786]}\end{array}$ & $\begin{array}{l}-0.00572 \\
{[0.00781]}\end{array}$ \\
\hline Bargain* & & & & & & & & & & \\
\hline Unavailability & $\begin{array}{l}0.000871 \\
{[0.00142]}\end{array}$ & $\begin{array}{l}-0.0106^{* *} \\
{[0.00477]}\end{array}$ & $\begin{array}{l}-0.00101 \\
{[0.00206]}\end{array}$ & $\begin{array}{c}0.00478 \\
{[0.00619]}\end{array}$ & $\begin{array}{c}0.00977 \\
{[0.00771]}\end{array}$ & $\begin{array}{l}-0.00161 \\
{[0.00921]}\end{array}$ & $\begin{array}{c}0.00297 \\
{[0.00294]}\end{array}$ & $\begin{array}{l}-0.00158 \\
{[0.00165]}\end{array}$ & $\begin{array}{c}-0.0072 \\
{[0.00760]}\end{array}$ & $\begin{array}{c}0.00354 \\
{[0.00796]}\end{array}$ \\
\hline Constant & $\begin{array}{c}0.0199 * * * \\
{[0.00140]}\end{array}$ & $\begin{array}{c}0.0256 * * * \\
{[0.00341]}\end{array}$ & $\begin{array}{c}0.0154 * * * \\
{[0.00202]}\end{array}$ & $\begin{array}{c}0.0522 * * * \\
{[0.00497]}\end{array}$ & $\begin{array}{l}0.177 * * * \\
{[0.00432]}\end{array}$ & $\begin{array}{c}0.0349 * * * \\
{[0.00511]}\end{array}$ & $\begin{array}{c}0.0804 * * * \\
{[0.00242]}\end{array}$ & $\begin{array}{c}0.00316^{* * *} \\
{[0.00100]}\end{array}$ & $\begin{array}{l}0.123 * * * \\
{[0.00613]}\end{array}$ & $\begin{array}{c}0.0831 * * * \\
{[0.00617]}\end{array}$ \\
\hline Observations & 6,069 & 6,069 & 6,069 & 6,069 & 6,069 & 6,069 & 6,069 & 6,069 & 6,069 & 6,069 \\
\hline R-squared & 0.02 & 0.04 & 0.007 & 0.062 & 0.112 & 0.069 & 0.034 & 0.043 & 0.046 & 0.01 \\
\hline
\end{tabular}

